\title{
Cloud, grid, P2P and internet computing: Recent trends and future directions
}

\author{
Sang-Soo Yeo ${ }^{1} \cdot$ Yu Chen ${ }^{2} \cdot$ Cho-Li Wang ${ }^{3}$
}

Published online: 20 March 2015

(C) Springer Science+Business Media New York 2015

\section{Introduction}

During the last decades, many researchers in cloud, grid, P2P and Internet computing community have been devoted to develop new and efficient algorithms and architectures. These technologies have been emerging as new paradigms for solving complex problems by enabling large-scale aggregation and sharing of computational resources and data. The effort has resulted in advances in this new paradigm for high performance computing systems. Nevertheless, there are still many research issues need to be addressed in the area of cloud, grid, P2P and Internet computing. This Special Issue aims at providing a forum to bring together researchers for sharing and exchanging cloud, grid, P2P and Internet computing related research, technologies, experience, and lessons for building clouds and grids of interoperability and coordination capabilities and services. We believe this SI will help the research community to inspire their novel ideas in the current state of cloud-grid-P2P interoperability issues. In addition, the SI will help to define the future goals and services that cloud and grid need to support in various intensive commercial and enterprise applications.

Sang-Soo Yeo

sangsooyeo@gmail.com

Yu Chen

ychen@binghamton.edu

Cho-Li Wang

choliwang@gmail.com

1 Mokwon University, Daejeon 302-729, South Korea

2 State University of New York - Binghamton, Binghamton, NY 13902, USA

3 University of Hong Kong, Hong Kong, China
We have received many manuscripts and each manuscript was blindly reviewed by at least three reviewers consisting of guest editors and external reviewers. After the first and second review processes, ten manuscripts were finally selected to be included in this special issue.

In Section 2, we present the summaries of the manuscript accepted in this issue. In the last section, we conclude with some observations and future work.

\section{Related work}

The first paper entitled "Dependability and reliability analysis of intra cluster routing technique" by Jan et al. [1], in which various routing schemes for small size cluster have been implemented and evaulated. They have considered different environmental conditions such as temperature, sound, vibration, pressure or motion by means of wireless sensor networks (WSNs), these nodes in WSNs are battery operated and therefore power management plays an important role in overall reliability. To achieve this task they suggested a hybrid intra cluster routing technique and load balancing scheme after analyzing various intra clustering methods.

The second paper entitled "Peer-to-peer usage analysis in dynamic databases," by Lin et al. [2], presented a maintenance fast updated high utility pattern tree for transaction modification (FUP-HUP-tree-MOD) algorithm for maintaining and updating the discovered high-utility itemsets (HUIs) with transaction modification. A built HUP tree can be also used as an effective structure to reduce the multiple database scan by keeping the necessary information for later maintenance and mining process. Based on the proposed algorithm with the built HUP tree, it is unnecessary to rescan the original database each time especially when the number of modified transactions is quiet small. Experimental results showed that 
their proposed algorithm has better performance compared to the traditional batch-mode approaches.

Another paper entitled "An efficient peer-to-peer and distributed scheduling for cloud and grid computing," by Rho et al. [3], developed the distributed scheduling service framework based on the following features: i) The resource management system is distributed in a hierarchical peer-to-peer network using resource content information that is characterized by system properties; and ii) The peered network provides an efficient message routing mechanism to process expressive QoS queries for preventing message flooding and performance bottlenecks.

The fourth paper entitled "VoIP-aware Network Attack Detection based-on Statistic and Behavior of SIP Traffic," by Lee et al. [4], proposed schemes to detect VoIP network attacks (such as VoIP DoS and SPAM) which improved the efficacy of VoIP detection using a statistical learning-based VoIP DoS detection scheme and reduced the false positive rate of SPAM detection using enhanced analysis factors and strong conditions. The proposed scheme can be implemented as a module of inline-based IPS systems and can work as a practical VoIPaware network security solution.

Fifth paper entitled "On the Security of a Certificateless online/offline signcryption for Internet of Things," by Shi et al. [5], proposed a certificate-less signcryption for the Internet of Things (IoT) environment. In this paper, authors have identified the vulnerability problems in private key compromised of earlier proposed scheme and designed their own new scheme which is resilient to various types of attacks in IoT environment. The performance analysis of the designed scheme proves the effectiveness of the proposed scheme.

Next paper entitled "Performance Evaluation for Delay Time Estimation in IEEE $802.16 \mathrm{~m}$ Sleep Mode," by Lee et al. [6], evaluated the delay time estimation in the sleep mode of IEEE $802.16 \mathrm{~m}$ by using M/D/1 queueing system with vacations and simulations under various traffic models. Their research offered solution to calculate the delay time with Poisson arrivals and experimental results to find parameters of power saving mechanisms based on arriving packet characteristic. The paper verified that both average sleep cycle and listening window are more dominant factors in terms of the delay time than traffic patterns.

Seventh paper entitled "Cost and Performance Effective Data Center Selection System for Scientific Federated Cloud," by Jaikar and Noh [7], experimentally studied the Scientific Federated Cloud by considering cost and performance of the data center. This paper is to select best data center with the help of network delay, cost, performance and availability of the data center. This paper follows the centralized management approach to solve the problem. It has verified that the proposed algorithm is very effective to choose the effective data center which is verified by simulator.
Eighth paper entitled "An Improved authentication Protocol for Session Initiation Protocol using Smart Card," by Tu et al. [8], proposed an improved session initiation protocol using smart card. Authors have identified the major drawback as impersonation attack in the existing scheme and designed a new scheme for session initiation using smart card for mitigation of impersonation attack. The proposed scheme has less computation cost and lower complexity as compared to the existing scheme. Also, a detailed security analysis of the proposed scheme is provided with respect to various types of attacks with respect to which the proposed scheme was found to be more secure than existing scheme.

Next paper entitled "A Granular Approach for UserCentric Network Analysis to Identify Digital Evidence," by Yasin et al. [9], revealed that the use of communication technology for social interaction through instant messenger has its own importance and significance over other channels such as cell phones and text messages in the aspect of digital forensic investigation. This paper also studied traits of instant messages after analyzing a collection of dataset over the period 2 years and 4-months, containing the MSN chat logs of single machine using third party Messenger Plus! application.

The last paper entitled "Peer-to-peer Aggregation for Dynamic Adjustments in Power Demand," by Pournaras [10], studied peer-to-peer aggregation in the application domain of Smart Grids and energy management. The ALMA mechanism is introduced that performs adjustments in power demand by making selections between different incentivized demand-options. Selections were based on aggregate consumption information provided by peer-to-peer aggregation mechanisms. The feasibility of dynamic adjustment in power demand was evaluated and confirmed using data from the current reality and practice of Smart Grids. This article appears in a previous issue of the journal: Volume 8, No. 2, March 2015.

\section{Conclusion}

Finally, our special thanks go to Prof. Xuemin Shen and all editorial staffs for their valuable supports throughout the preparation and publication of this special issue. We would like to thank all authors for their contributions to this special issue. We also extend our thanks to the following external reviewers for their excellent help in reviewing the manuscripts.

\section{References}

1. Jan H, Paul A, Minhas AA, Ahmad A, Jabbar S, Kim M (2014) Dependability and reliability analysis of intra cluster routing technique. Peer-to-Peer Netw Appl. doi:10.1007/s12083-014-0311-1 
2. Lin C-W, Gan W, Hong T-P, Park SO, Chen B-W (2014) Peer-topeer usage analysis in dynamic databases. Peer-to-Peer Netw Appl. doi:10.1007/s12083-014-0290-2

3. Rho S, Chang H, Kim S, Lee YS (2014) An efficient peer-to-peer and distributed scheduling for cloud and grid computing. Peer-toPeer Netw Appl. doi:10.1007/s12083-014-0270-6

4. Lee J, Cho K, Lee CY, Kim S (2014) VoIP-aware network attack detection based-on statistic and behavior of SIP traffic. Peer-to-Peer Netw Appl. doi:10.1007/s12083-014-0289-8

5. Shi W, Kumar N, Gong P, Chilamkurti N, Chang H (2014) On the security of a certificateless online/offline signcryption for internet of things. Peer-to-Peer Netw Appl. doi:10.1007/s12083-014-02493

6. Lee KH, Choi JY, Ma JS (2014) Performance evaluation for delay time estimation in IEEE $802.16 \mathrm{~m}$ sleep mode. Peer-to-Peer Netw Appl. doi:10.1007/s12083-014-0262-6

7. Jaikar A, Noh S-Y (2014) Cost and performance effective data center selection system for scientific federated cloud. Peer-to-Peer Netw Appl. doi:10.1007/s12083-014-0261-7

8. Tu H, Kumar N, Chilamkurti N, Rho S (2014) An improved authentication protocol for session initiation protocol using smart card. Peer-to-Peer Netw Appl. doi:10.1007/s12083-014-0248-4

9. Yasin M, Qureshi JA, Kausar F, Kim J, Seo J (2014) A granular approach for user-centric network analysis to identify digital evidence. Peer-to-Peer Netw Appl. doi:10.1007/s12083-014-0250-x

10. Pournaras E, Warnier M, Brazier FMT (2014) Peer-to-peer aggregation for dynamic adjustments in power demand. Peer-to-Peer Netw Appl. doi:10.1007/s12083-013-0246-y

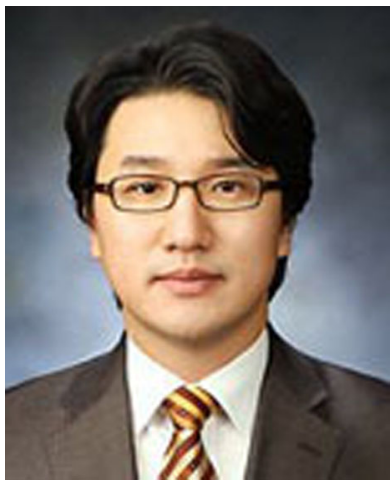

Dr. Sang-Soo Yeo received his master's degree and Ph.D degree in Computer Science \& Engineering from Chung-Ang University, Seoul, Korea. He was a visiting scholar at Kyushu University, Japan. He worked for BTWorks, Inc. as a General Manager. He is currently a professor at the Division of Convergence Computer \& Media Engineering, Mokwon University, Daejeon, Korea. He is President of the Institution of Creative Research Professionals (ICRP), and Vice President of ICT Platform Society. He has been serving as chairs in many conferences and workshops. Dr. Yeo's research interests include Security, Ubiquitous Computing, Multimedia Service, Ubiquitous Computing, Embedded System, and Bioinformatics. He is a member of the KIPS, KIISE, KICS, KIISC, KMS, KDAS, KONI, KIMICS, KIIT, IEEE, IEEE CS, and IEEE ComSoc.

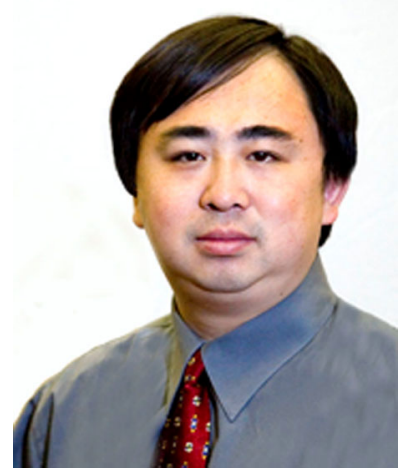

Dr. Yu Chen is an Associate Professor of Electrical and Computer Engineering at the State University of New York - Binghamton. He received the Ph.D. in Electrical Engineering from the University of Southern California (USC) in 2006 under the supervision of Professor Kai Hwang. Before join Professor Hwang's group, he was working as a research assistant in the Advanced Interconnection and Network Technology Lab led by Professor Anthony F. J. Levi. His research has been funded by NSF, DoD, AFOSR, AFRL, New York State, and industrial partners. He has authored or coauthored more than 90 scientific papers in refereed journals, conferences, and book chapters. He is a member of ACM, IEEE (Computer \& Communication Societies) and SPIE.

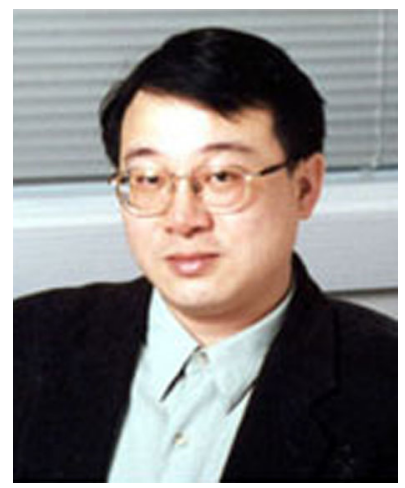

Dr. Cho-Li Wang received his $\mathrm{Ph} . \mathrm{D}$. degree from University of Southern California in 1995 . He is currently a professor at the Department of Computer Science at The University of Hong Kong. His research mainly focuses on the system softwares for supporting Manycore/GPU, Cloud and Big Data computing. Dr. Wang has published more than 150 papers in various peer reviewed journals and conference proceedings. He is/was on the editorial boards of several international journals, including IEEE Transactions on Computers (20062010), IEEE Transactions on Cloud Computing (2013-), and Journal of Information Science and Engineering (2009-). 